\title{
Frequency-tunable microwave field detection in an atomic vapor cell
}

Andrew Horsley and Philipp Treutlein

Citation: Appl. Phys. Lett. 108, 211102 (2016); doi: 10.1063/1.4950805

View online: http://dx.doi.org/10.1063/1.4950805

View Table of Contents: http://aip.scitation.org/toc/apl/108/21

Published by the American Institute of Physics

\section{Articles you may be interested in}

Simple microwave field imaging technique using hot atomic vapor cells

Appl. Phys. Lett. 101, 181107181107 (2012); 10.1063/1.4760267

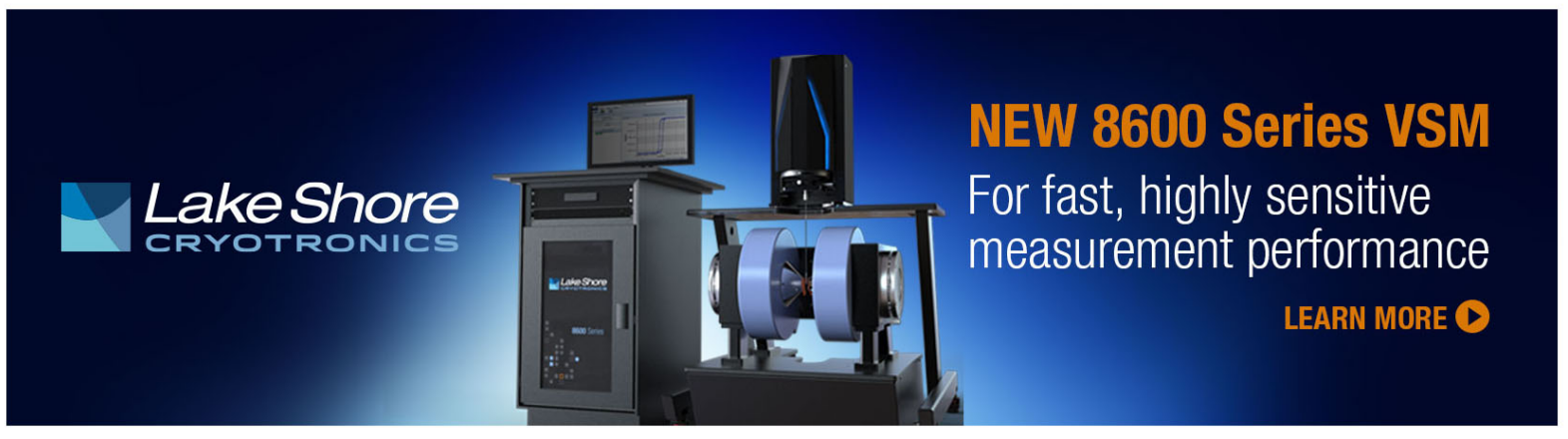




\title{
Frequency-tunable microwave field detection in an atomic vapor cell
}

\author{
Andrew Horsley a) and Philipp Treutlein ${ }^{\text {b) }}$ \\ Departement Physik, Universität Basel, CH-4056 Basel, Switzerland
}

(Received 26 February 2016; accepted 5 May 2016; published online 23 May 2016)

\begin{abstract}
We use an atomic vapor cell as a frequency tunable microwave field detector operating at frequencies from $\mathrm{GHz}$ to tens of $\mathrm{GHz}$. We detect microwave magnetic fields from $2.3 \mathrm{GHz}$ to $26.4 \mathrm{GHz}$, and measure the amplitude of the $\sigma_{+}$component of an $18 \mathrm{GHz}$ microwave field. Our proof-of-principle demonstration represents a four orders of magnitude extension of the frequency tunable range of atomic magnetometers from their previous dc to several $\mathrm{MHz}$ range. When integrated with a high-resolution microwave imaging system [Horsley et al., New J. Phys. 17, 112002 (2015)], this will allow for the complete reconstruction of the vector components of a microwave magnetic field and the relative phase between them. Potential applications include near-field characterisation of microwave circuitry and devices, and medical microwave sensing and imaging. Published by AIP Publishing. [http://dx.doi.org/10.1063/1.4950805]
\end{abstract}

Atomic vapor cell magnetometers are among the most sensitive detectors for magnetic fields $\mathrm{s}^{2-4}$ and show promise in applications including gyroscopes, ${ }^{5}$ explosives detection, ${ }^{6}$ materials characterization, ${ }^{7-9}$ in MRI for both medical ${ }^{10}$ and microfluidics applications, ${ }^{11}$ and for magnetic imaging of the human heart ${ }^{12,13}$ and brain. ${ }^{14-17}$ Tunable atomic magnetometers have previously operated from dc to rf of a few MHz. In this letter, we demonstrate the principle of a continuously frequency tunable microwave magnetometer, capable of detecting magnetic fields from $\mathrm{GHz}$ to tens of $\mathrm{GHz}$ frequencies. This represents a four orders of magnitude extension of the atomic magnetometer frequency tunable range. Potential applications include near-field characterisation of microwave circuitry, ${ }^{1,18-20}$ and medical microwave sensing and imaging, ${ }^{21-23}$ where high resolution and intrinsically calibrated atomic sensors could replace bulky and fieldperturbing antennas.

At the fixed microwave frequency of $6.8 \mathrm{GHz}$, we have previously used atoms to produce polarisation-resolved images of microwave magnetic near-field distributions. ${ }^{1,24-27}$ We have achieved $50 \times 50 \times 140 \mu \mathrm{m}^{3}$ spatial resolution and $1 \mu \mathrm{T} / \mathrm{Hz}^{-1 / 2}$ sensitivity, observing good agreement between the measured fields and simulations for simple test structures. ${ }^{1}$ (Atom-like) nitrogen vacancy (NV) centres have recently been used for nanoscale near-field detection ${ }^{28}$ and imaging, ${ }^{29}$ and microwave electric field imaging has also been demonstrated at discrete frequencies using Rydberg atoms. ${ }^{30-33}$ To-date, however, there has been no atomic sensor available for the detection of fields at arbitrary microwave frequencies, as required for most applications.

Our frequency-tunable sensing scheme is able to operate in either of two modes: to detect microwaves of unknown frequency (i.e., as a microwave spectrum analyser); or to accurately measure the amplitudes of microwave magnetic fields $\left(\mathrm{B}_{\mathrm{mw}}\right)$ of known frequency. In this letter, we focus on the latter, measuring $B_{\mathrm{mw}}$ through coherent

\footnotetext{
a)andrew.horsley@unibas.ch

b) philipp.treutlein@unibas.ch
}

Rabi oscillations driven by the microwave on atomic hyperfine transitions. ${ }^{24,25}$ The atomic transitions are sensitive to a narrow microwave frequency band, and we use a dc magnetic field $\left(\mathrm{B}_{\mathrm{dc}}\right)$ to tune the transition frequency to the desired value. This is conceptually similar to rf magnetometry, where a dc magnetic field is used to tune the Zeeman splitting of adjacent $\mathrm{m}_{F}$ states. ${ }^{3}$ However, much larger dc fields are required for microwave frequencies, bringing us into the hyperfine Paschen-Back regime. Moreover, since our technique is based on measurements of frequency rather than amplitude, it is intrinsically calibrated and less sensitive to environmental noise. In this letter, we work with the ${ }^{87} \mathrm{Rb}$ hyperfine ground states; however, our technique is applicable to any microwave magnetic dipole transition with optical read-out of one of the states, such as other alkali atoms and $\mathrm{NV}$ centres.

Figure 1 shows the splitting of the ${ }^{87} \mathrm{Rb} 5 \mathrm{~S}_{1 / 2}$ ground state in an applied dc magnetic field, calculated using the Breit-Rabi formula. ${ }^{34}$ We label the 8 hyperfine states $A_{1} \rightarrow A_{8}$, in order of increasing energy. At $B_{d c}=0$, the states are grouped according to the $\left|F, m_{F}\right\rangle$ basis defined by the atomic Hamiltonian, and split by $6.8 \mathrm{GHz}$. As $\mathrm{B}_{\mathrm{dc}}$ is increased, the hyperfine states move towards the $\left|I, m_{I}, J, m_{J}\right\rangle$ basis defined by the dc field. This is accompanied by a corresponding shift in transition frequencies and coupling strengths between the hyperfine states. An applied microwave field will drive Rabi oscillations on a given transition that has been tuned to resonance. The amplitude of the microwave vector component $B_{\gamma}$ is given by the measured Rabi oscillation frequency, $\Omega_{\mathrm{R}}$, the transition matrix element, and well-known fundamental constants. For a microwave resonant with transition $\mathrm{T}_{i f}$, we have

$$
B_{\gamma}=\left|\alpha_{i f}\right| \frac{\hbar}{\mu_{B}}\left|\Omega_{\mathrm{R}}\right|,
$$

where $\gamma=-, \pi,+$ is the polarisation of the transition, and

$$
\alpha_{i f} \equiv \frac{1}{2\left\langle A_{f}\left|J_{\gamma}\right| A_{i}\right\rangle} .
$$




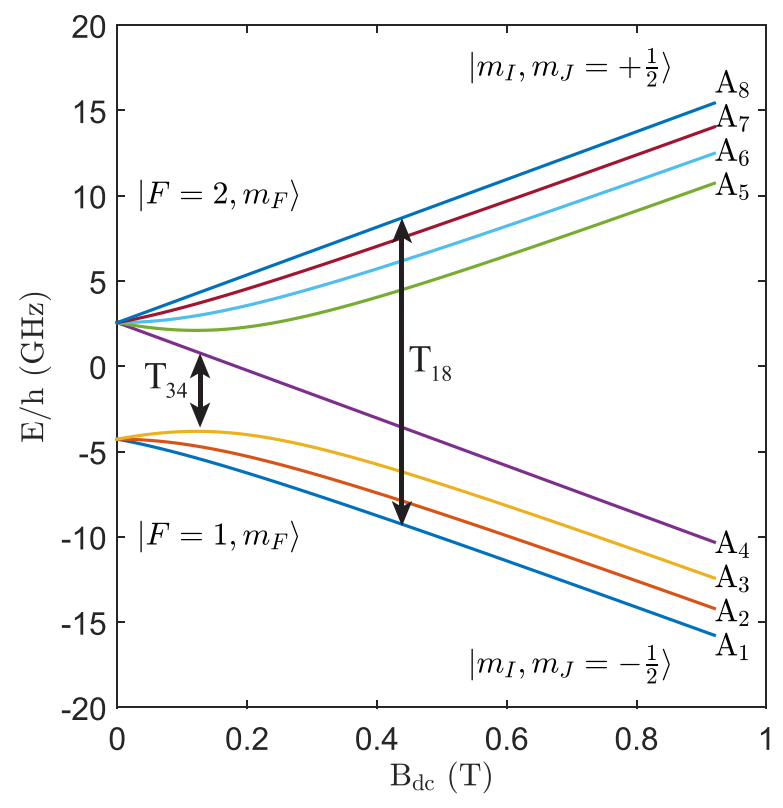

FIG. 1. Energy splitting of the ${ }^{87} \mathrm{Rb}$ hyperfine ground state levels as a function of applied dc magnetic field. Arrows indicate the hyperfine transitions used in this work.

The coupling factor $\alpha_{i f}$ is a function of the applied dc magnetic field. As $\mathrm{B}_{\mathrm{dc}}$ increases, $\alpha \rightarrow 1$ for the $\sigma_{+}$polarised transitions, and $\alpha \rightarrow 0$ for the $\sigma_{-}$and $\pi$ transitions. For ${ }^{87} \mathrm{Rb}$, we can find $\sigma_{-}(\pi)$ transitions where $|\alpha|>0.15$ for microwave frequencies within the range $2.2-9.1 \mathrm{GHz}(5.9-22.8 \mathrm{GHz}){ }^{35}$ Strong $\sigma_{+}$transitions are available for all frequencies. Previous $\mathrm{B}_{\mathrm{mw}}$ reconstruction schemes have relied on the presence of strong $\pi$ transitions. ${ }^{1}$ However, it is possible to completely reconstruct the vector components of the microwave magnetic field amplitude, and the relative phases between them, using only $\sigma_{+}$ transitions. ${ }^{35}$ The reconstruction then requires measurements of $\mathrm{B}_{+}$with the dc magnetic field both parallel and anti-parallel to each of the lab-frame $x, y$, and $z$ axes. In this letter, we address two microwave transitions: the $\sigma_{+}$polarised $\mathrm{T}_{18}$ transition and the $\sigma_{-}$polarised $\mathrm{T}_{34}$ transition.

We use a $2 \mathrm{~mm}$ thick, microfabricated vapor cell (Fig. 2(a)), filled with natural $\mathrm{Rb}$ and 63 mbar of $\mathrm{N}_{2}$ buffer gas. ${ }^{26,36}$ Doppler and collisional broadening result in an optical linewidth of $1.5 \mathrm{GHz}$. We use a $780 \mathrm{~nm}$ laser, linearly polarised along $\mathrm{B}_{\mathrm{dc}}$, for both optical pumping and probing. A water-cooled solenoid ${ }^{37}$ provides dc magnetic fields up to $0.8 \mathrm{~T}$ (Fig. 2(b)). We heat the vapor cell using a $2 \mathrm{~W}$ laser at $1500 \mathrm{~nm}^{35,38}$ and obtain the cell temperature by fitting optical absorption spectra (taken at $\mathrm{B}_{\mathrm{dc}}=0$ ) with the model described in Ref. 39.

We can detect microwaves over a broad range of frequencies. Figure 3(a) shows experimentally obtained doubleresonance (DR) peaks for microwaves spanning frequencies from $2.3 \mathrm{GHz}$ to $26.4 \mathrm{GHz}$. The laser beam is applied continuously, and its frequency is tuned to resonance with one of the hyperfine states. This partially depopulates the state through optical pumping. Through the transmission of the laser onto a photodiode, we monitor the change in optical density $\left(\mathrm{OD}_{\mathrm{mw}}\right)$ as we sweep the frequency of an applied microwave field. Whenever the microwave frequency crosses a hyperfine transition coupling to the optically pumped state, we see a peak in $\mathrm{OD}_{\mathrm{mw}}$, corresponding to repopulation of the state. Each peak in Fig. 3 represents a separate measurement, with $B_{d c}$ changed between measurements. Peaks in the range $7-26.4 \mathrm{GHz}$ (red) were measured on the $\sigma_{+} \mathrm{T}_{18}$ transition, while peaks in the range $2.3-6 \mathrm{GHz}$ (blue) were measured on the $\sigma_{-} \mathrm{T}_{34}$ transition. The laser beam probed the approximate spatial centre of the dc magnetic field (see Fig. 4(b)), with inhomogeneities in the dc field resulting in a $1 \mathrm{MHz}$ (FWHM) DR linewidth. The peak area is proportional to $\Omega_{R}$; however, it is also

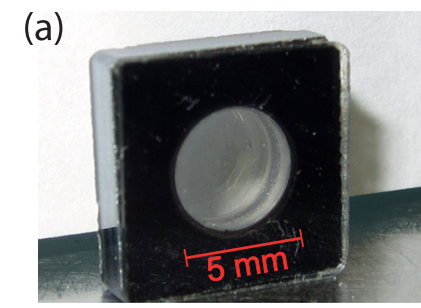

(b)



(d) (c)

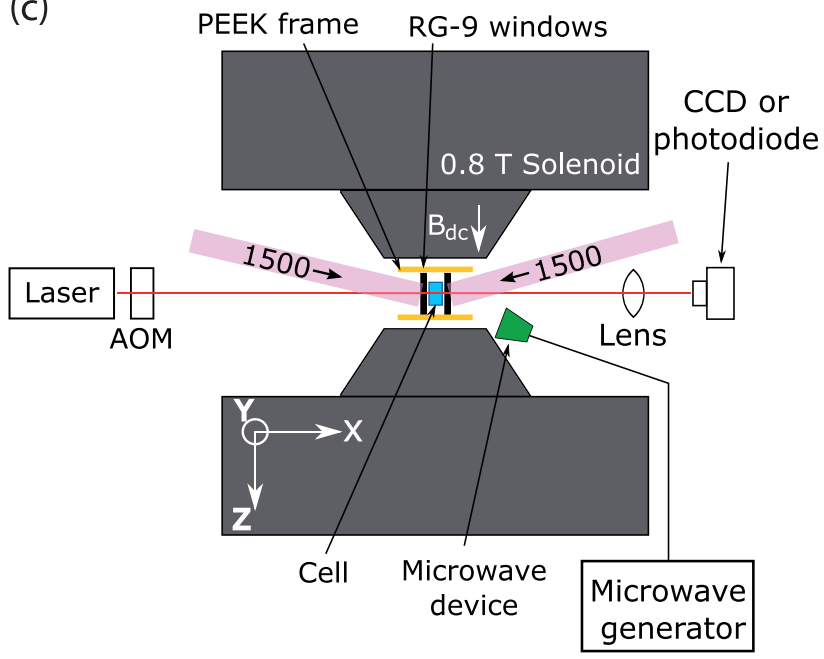

probe

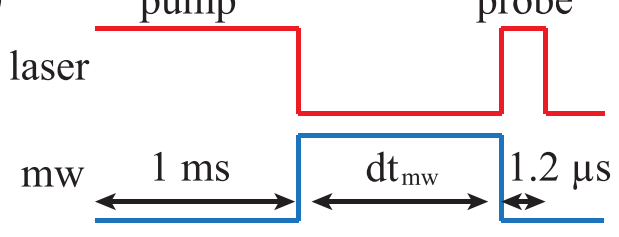

FIG. 2. (a) Vapor cell; (b) the experiment setup, showing the vapor cell sandwiched between two RG-9 glass pieces and mounted on the PEEK frame inside the solenoid. The gold microwave output coupler can be seen above the cell; (c) schematic of the experiment setup; (d) the Rabi sequence used to obtain microwave amplitudes. 
(a)

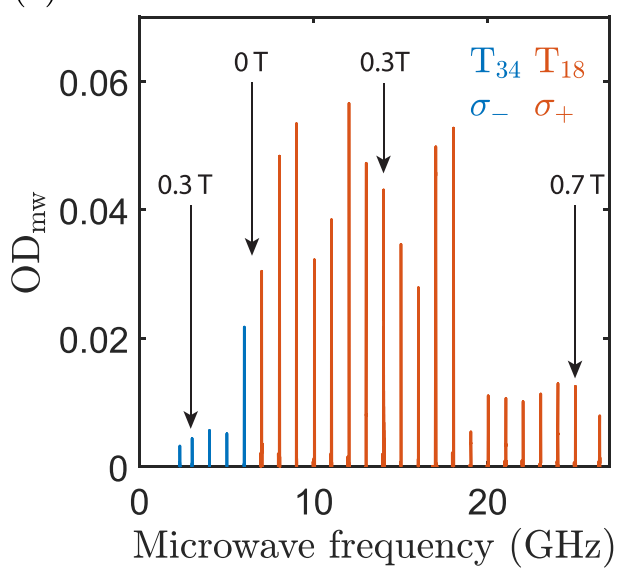

(b)

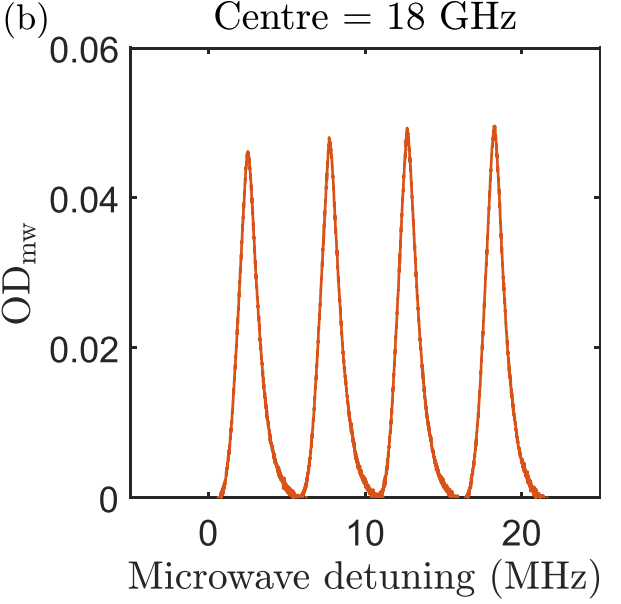

FIG. 3. Detection of microwave signals at arbitrary frequencies. (a) DR detection of frequencies ranging from $2.3 \mathrm{GHz}$ to $26.4 \mathrm{GHz}$. Each peak corresponds to a single experimental run taken using a different $\mathrm{B}_{\mathrm{dc}}$. The data were taken on both the $\mathrm{T}_{34}$ (blue) and $\mathrm{T}_{18}$ (red) transitions, and show the change in optical density induced by the microwave. Labels in black indicate relevant $B_{\mathrm{dc}}$ strengths. (b) DR peaks for frequencies near $18 \mathrm{GHz}$, showing fine-tuning of the detection frequency by adjusting $B_{\mathrm{dc}}$. sensitive to fluctuations in the laser intensity and cell temperature, and calibration with a known field is required to extract $\Omega_{R}$. For fixed conditions (not shown), we observe the expected linear scaling of the peak area with the square-root of the microwave power. The lower limit on microwave frequency in Fig. 3(a) was imposed by the asymptotic behaviour of the $\mathrm{T}_{34}$ transition frequency at high $\mathrm{B}_{\mathrm{dc}}{ }^{35}$ Other transitions (e.g., $\mathrm{T}_{45}$ ) are available with frequencies down to dc, in which case the lower detection limit will be given by the optical distinguishability of the neighbouring hyperfine states, in general on the order of the $0.5 \mathrm{GHz}$ Doppler broadening in a cell without buffer gas. The upper frequency limit is given only by technical limitations, e.g., the available $\mathrm{B}_{\mathrm{dc}}$ strength, or in our case, the microwave frequency generator.
The dc magnetic field provides fine control over the microwave detection frequency, as shown in Fig. 3(b). Each DR peak represents a single measurement on the $\mathrm{T}_{18}$ transition, with the zero of the horizontal axis corresponding to a microwave frequency of $18 \mathrm{GHz}$. By scanning $\mathrm{B}_{\mathrm{dc}}$, such DR measurements could be used in a spectrum analyser mode, to search for microwave fields of unknown frequency.

For an accurate measure of the $\mathrm{B}_{\mathrm{mw}}$ amplitude, we need to measure the Rabi frequency directly. We demonstrate this by measuring the $\sigma_{+}$amplitude of an $18 \mathrm{GHz}$ microwave. The observed oscillations are a function of both microwave amplitude $\left(\propto \Omega_{R}\right)$ and detuning from resonance $(\Delta)$, with a frequency $\Omega=\sqrt{\Omega_{R}^{2}+\Delta^{2}}$. In general, it is simple to directly
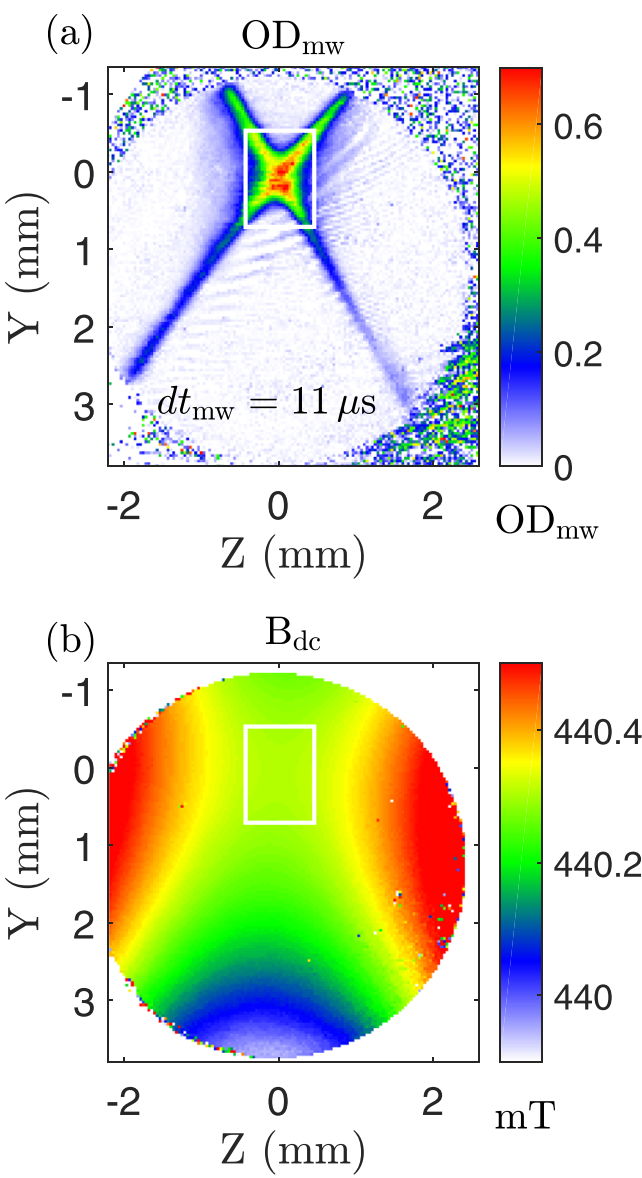

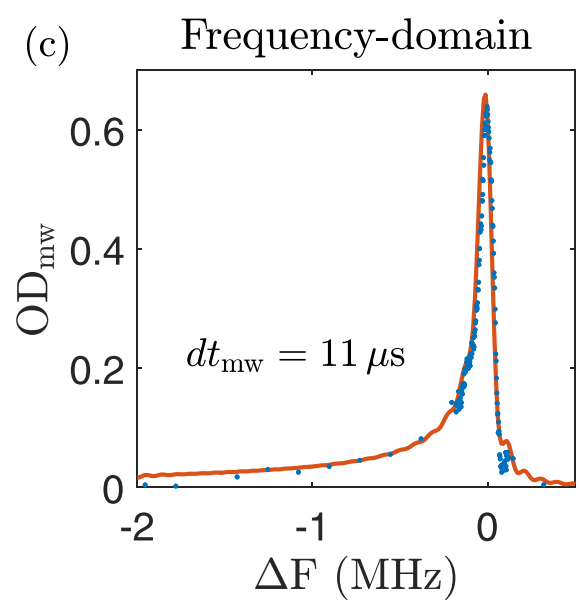

(d)

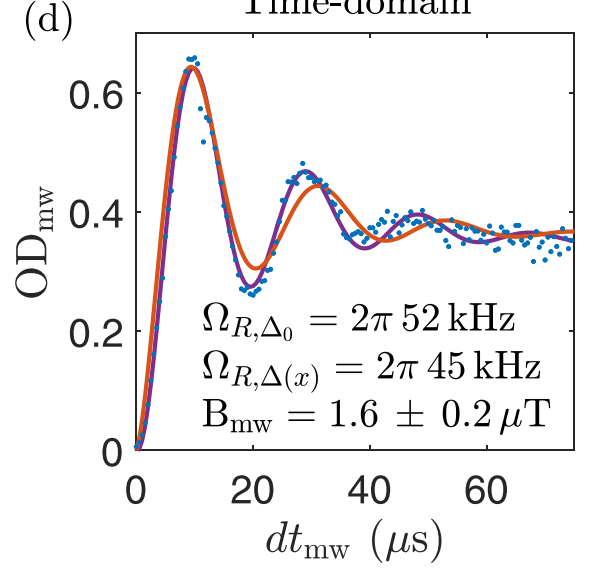

FIG. 4. Measuring an 18.003 GHz microwave field amplitude. Experimentally obtained images of (a) $\mathrm{OD}_{\mathrm{mw}}$ for a microwave pulse resonant with the $\mathrm{T}_{18}$ transition in the $\mathrm{B}_{\mathrm{dc}}$ field centre; (b) map of the local $B_{\mathrm{dc}}$ field extracted from measurements as in (a). The circular field of view is given by the cell walls, and the white boxes indicate the region appropriate for microwave sensing; (c) and (d) scans in the frequency and time domains, for the pixel at $(z=0 \mathrm{~mm}, y=0 \mathrm{~mm})$. Fits are shown assuming a constant microwave detuning along the $x$-axis (purple, time domain only, $\Omega_{\mathrm{R}, \Delta_{0}}$ ), and allowing for an inhomogeneous detuning (red, simultaneous fit to time and frequency domain data, $\Omega_{\mathrm{R}, \Delta(x)}$. 
measure the detuning, e.g., from the peak of a DR scan. We use a relatively thick cell in our proof-of-principle setup, however, and must account for a non-negligible $\mathrm{B}_{\mathrm{dc}}$ inhomogeneity along the $x$-axis, which the laser integrates over as it passes through the cell. Our experiment "Rabi" sequence, shown schematically in Fig. 2(d), begins with an optical pumping pulse to depopulate the $A_{1}$ state. A microwave pulse of duration $d t_{\mathrm{mw}}$ then drives Rabi oscillations between the $\mathrm{A}_{1}$ and $\mathrm{A}_{8}$ states, and we detect the repopulation of the $\mathrm{A}_{1}$ state (i.e., $\mathrm{OD}_{\mathrm{mw}}$ ) with a $1.2 \mu$ s probe laser pulse. We repeat this sequence, scanning either the frequency (Fig. 4(c)) or duration (Fig. 4(d)) of the microwave pulse. The frequency domain scan primarily constrains $B_{d c}$ (Fig. 4(b)), and we extract the $\mathrm{B}_{\mathrm{mw}}$ amplitude by simultaneously fitting the two scans. ${ }^{35}$ In future setups, the frequency domain scan will be unnecessary.

We used the $T_{18}$ transition for sensing, with the frequency of the laser tuned to address the $\mathrm{A}_{1}$ level. A solenoid field of $\mathrm{B}_{\mathrm{dc}} \approx 0.44 \mathrm{~T}$ tuned the $\mathrm{T}_{18}$ transition of atoms in the $B_{\mathrm{dc}}$ field centre to resonance with the applied $18 \mathrm{GHz}$ microwave. At this dc field, the $A_{1}$ level is optically wellresolved ${ }^{40}$ and almost all of the measured optical density of $\mathrm{OD}=2.1$ was due to atoms in the $\mathrm{A}_{1}$ state. The frequency domain scan was performed around $18.003 \mathrm{GHz}$ with a fixed microwave pulse duration of $d t_{\mathrm{mw}}=11 \mu \mathrm{s}$, corresponding roughly to a $\pi$ pulse in the centre of the $\mathrm{B}_{\mathrm{dc}}$ field, while the time domain scan was performed with a fixed frequency of $18.003 \mathrm{GHz}$.

We are working towards frequency tunable microwave imaging, and therefore use a CCD camera and the technique of absorption imaging for detection. ${ }^{1,40,41}$ The laser illuminates the entire cell, and we obtain images of $\mathrm{OD}_{\mathrm{mw}}$ (Fig. 4(a)). Due to inhomogeneities in the $\mathrm{B}_{\mathrm{dc}}$ field, we only present microwave detection for a single pixel near the $B_{d c}$ centre. If only single-channel sensing is required, real-time monitoring of the Rabi oscillations should also be possible using a photodiode and an off-resonant probe laser. The atomic response traced a contour line of the $\mathrm{B}_{\mathrm{dc}}$ field in the cell, as shown in Fig. 4(a), which shows an image of $\mathrm{OD}_{\mathrm{mw}}$ for an $11 \mu$ s long $18.003 \mathrm{GHz}$ microwave pulse. Scanning the microwave frequency (Fig. 4(c)), we could quickly estimate the local $\mathrm{T}_{18}$ transition frequency from the peak in $\mathrm{OD}_{\mathrm{mw}}$. Using the Breit-Rabi formula, this transition frequency yielded an image of the applied $\mathrm{B}_{\mathrm{dc}}$, shown in Fig. 4(b). We see that the solenoid field is saddle-shaped, homogeneous to better than $10^{-3}$, but nonetheless varying by several Gauss over the cell. The plane of the image is slightly offset from the $x=0$ centre of the solenoid field.

The simultaneous fit of the Figs. 4(c) and 4(d) frequency and time domain data, described in detail in the supplementary material, yielded a Rabi frequency of $\Omega_{\mathrm{R}, \Delta(x)}=2 \pi 45$ $\mathrm{kHz}$, corresponding to $\mathrm{B}_{\mathrm{mw}}=1.6 \mu \mathrm{T}$. The shape of the frequency scan data in Fig. 4(c) is primarily determined by the variation in $\mathrm{B}_{\mathrm{dc}}$ along the $x$-axis, and is well-matched by the fit. The peak corresponds to atoms around the $\mathrm{B}_{\mathrm{dc}}$ centre, where the field is relatively flat, while the long tail is due to the drop-off of $\mathrm{B}_{\mathrm{dc}}$ away from the centre. The time domain fit in Fig. 4(d) underestimates the oscillation frequency, due to constraints imposed by the simultaneous fitting of time and frequency domain data with our relatively simple model. In fact, we find much better agreement with the time domain data if we simply assume a constant $\Delta_{0}=-4 \mathrm{kHz}$, given by the frequency domain peak position, and fit to the time domain data only. This is shown by the purple line in Fig. 4(d), and we obtain $\Omega_{\mathrm{R}, \Delta_{0}}=2 \pi 52 \mathrm{kHz}$, corresponding to $\mathrm{B}_{\mathrm{mw}}=1.8 \mu \mathrm{T}$. By neglecting the range of larger detunings along the $x$-axis, this value serves as an upper bound on $\mathrm{B}_{\mathrm{mw}}$. We can therefore estimate a $\mathrm{B}_{\mathrm{mw}}$ accuracy of $10 \%$ in our current proof-of-principle setup. We emphasise that in a high resolution setup using a thinner vapor cell, ${ }^{1}$ the image analysis will be much simpler, with $\mathrm{B}_{\mathrm{mw}}$ directly extracted from time domain data and accuracy on the $10^{-3}$ level.

In conclusion, we have presented a proof-of-principle demonstration of microwave magnetic field detection for microwaves of any frequency, obtaining the amplitude of the $\sigma_{+}$component of an $18 \mathrm{GHz}$ microwave field, and detecting magnetic fields at frequencies from $2.3 \mathrm{GHz}$ to $26.4 \mathrm{GHz}$. Magnetic field detection of frequencies up to $50 \mathrm{GHz}$ should be achievable with room-temperature iron-bore solenoids (which can operate to $\sim 1.6 \mathrm{~T}$ ), and given sufficient dc field homogeneity, it may be possible to detect fields up to $1 \mathrm{THz}$ using the strongest available superconducting solenoids ( $>35 \mathrm{~T}$ ). It would be interesting to explore the frequency tunability presented here with other detection techniques, such as Faraday rotation or EIT, and with other systems, such as NV centres. ${ }^{29}$

Our frequency tunable detection provides an essential step towards an atom-based characterisation tool for microwave devices operating at any frequency. To realise this in practical form, the frequency tunability demonstrated in this letter should now be integrated with a high resolution imaging setup. ${ }^{1}$ We note, however, that for some devices, such as circulators, the large applied $\mathrm{B}_{\mathrm{dc}}$ fields may significantly perturb the device operation. High spatial resolution, provided by a thin vapor cell, will have the added benefit of significantly reducing our sensitivity to $\mathrm{B}_{\mathrm{dc}}$ inhomogeneities. For a $200 \mu \mathrm{m}$ thick cell operating in the $\mathrm{B}_{\mathrm{dc}}$ field shown in Fig. $4(\mathrm{~b}), \mathrm{B}_{\mathrm{dc}}$ variation along the $x$-axis should be negligible, on the order of $2 \pi \times 1 \mathrm{kHz}$. The transverse variation over a $50 \times$ $50 \mu \mathrm{m}^{2}$ pixel at the $\mathrm{B}_{\mathrm{dc}}$ centre would be $2 \pi \times 0.2 \mathrm{kHz}$, increasing to $2 \pi \times 80 \mathrm{kHz}$ at a distance $1 \mathrm{~mm}$ either side of the centre. This is entirely acceptable compared to the tens to hundreds of $\mathrm{kHz}$ Rabi frequencies observed in Ref. 1. Moreover, the $\mathrm{B}_{\mathrm{dc}}$ inhomogeneity could also be reduced by using shimming coils. We expect the sensitivity to be slightly better than for the fixed-frequency imaging reported in Ref. 1, due to the suppression of $\mathrm{Rb}$ spin-exchange relaxation in large $\mathrm{B}_{\mathrm{dc}}$ fields. ${ }^{42}$ The integrated setup could be miniaturised by replacing the bulky solenoid with permanent rare-earth magnets, with $\mathrm{B}_{\mathrm{dc}}$ adjusted through the magnet separation. ${ }^{43,44}$

This work was supported by the Swiss National Science Foundation (SNFS). We thank G. Mileti, C. Affolderbach, and Y. Pétremand for providing the vapor cell, and the group of P. Maletinsky for the loan of microwave circuitry. We also thank M. Belloni for interesting discussions, and C. Affolderbach for careful reading of the manuscript.

\footnotetext{
${ }^{1}$ A. Horsley, G.-X. Du, and P. Treutlein, New J. Phys. 17, 112002 (2015).

${ }^{2}$ D. Budker and M. Romalis, Nat. Phys. 3, 227 (2007).
} 
${ }^{3}$ I. M. Savukov, S. J. Seltzer, M. V. Romalis, and K. L. Sauer, Phys. Rev. Lett. 95, 063004 (2005).

${ }^{4}$ D. Sheng, S. Li, N. Dural, and M. V. Romalis, Phys. Rev. Lett. 110, 160802 (2013).

${ }^{5}$ E. A. Donley, J. L. Long, T. C. Liebisch, E. R. Hodby, T. A. Fisher, and J. Kitching, Phys. Rev. A 79, 013420 (2009).

${ }^{6}$ S.-K. Lee, K. L. Sauer, S. J. Seltzer, O. Alem, and M. V. Romalis, Appl. Phys. Lett. 89, 214106 (2006).

${ }^{7}$ M. V. Romalis and H. B. Dang, Mater. Today 14, 258 (2011).

${ }^{8}$ C. Deans, L. Marmugi, S. Hussain, and F. Renzoni, Appl. Phys. Lett. 108, 103503 (2016).

${ }^{9}$ A. Wickenbrock, N. Leefer, J. W. Blanchard, and D. Budker, Appl. Phys. Lett. 108, 183507 (2016).

${ }^{10}$ I. Savukov and T. Karaulanov, J. Mag. Res. 231, 39 (2013).

${ }^{11}$ S. Xu, C. W. Crawford, S. Rochester, V. Yashchuk, D. Budker, and A. Pines, Phys. Rev. A 78, 013404 (2008).

${ }^{12}$ G. Bison, N. Castagna, A. Hofer, P. Knowles, J.-L. Schenker, M. Kasprzak, H. Saudan, and A. Weis, Appl. Phys. Lett. 95, 173701 (2009).

${ }^{13}$ O. Alem, T. H. Sander, R. Mhaskar, J. LeBlanc, H. Eswaran, U. Steinhoff, Y. Okada, J. Kitching, L. Trahms, and S. Knappe, Phys. Med. Biol. 60, 4797 (2015).

${ }^{14}$ I. Savukov and T. Karaulanov, Appl. Phys. Lett. 103, 43703 (2013).

${ }^{15}$ C. N. Johnson, P. D. D. Schwindt, and M. Weisend, Phys. Med. Biol. 58, 6065 (2013).

${ }^{16}$ R. Wyllie, M. Kauer, R. T. Wakai, and T. G. Walker, Opt. Lett. 37, 2247 (2012).

${ }^{17}$ V. K. Shah and R. T. Wakai, Phys. Med. Biol. 58, 8153 (2013).

${ }^{18}$ S. Sayil, D. J. Kerns, and S. Kerns, IEEE Trans. Instrum. Meas. 54, 2082 (2005).

${ }^{19}$ T. Dubois, S. Jarrix, A. Penarier, P. Nouvel, D. Gasquet, L. Chusseau, and B. Azais, IEEE Trans. Instrum. Meas. 57, 2398 (2008).

${ }^{20}$ G. M. Sardi, A. Lucibello, M. Kasper, G. Gramse, E. Proietti, F. Kienberger, and R. Marcelli, Appl. Phys. Lett. 107, 033107 (2015).

${ }^{21}$ E. C. Fear, S. C. Hagness, P. M. Meaney, M. Okoniewski, and M. A. Stuchly, IEEE Microwave Mag. 3, 48 (2002).

${ }^{22}$ N. Nikolova, IEEE Microwave Mag. 12, 78 (2011).

${ }^{23}$ R. Chandra, H. Zhou, I. Balasingham, and R. M. Narayanan, IEEE Trans. Biomed. Eng. 62, 1667 (2015).

${ }^{24}$ P. Böhi, M. F. Riedel, T. W. Hänsch, and P. Treutlein, Appl. Phys. Lett. 97, 051101 (2010).
${ }^{25}$ P. Böhi and P. Treutlein, Appl. Phys. Lett. 101, 181107 (2012).

${ }^{26}$ A. Horsley, G.-X. Du, M. Pellaton, C. Affolderbach, G. Mileti, and P. Treutlein, Phys. Rev. A 88, 063407 (2013).

${ }^{27}$ C. Affolderbach, G.-X. Du, T. Bandi, A. Horsley, P. Treutlein, and G. Mileti, IEEE Trans. Instrum. Meas. 64, 3629 (2015).

${ }^{28}$ P. Wang, Z. Yuan, P. Huang, X. Rong, M. Wang, X. Xu, C. Duan, C. Ju, F. Shi, and J. Du, Nat. Commun. 6, 6631 (2015).

${ }^{29}$ P. Appel, M. Ganzhorn, E. Neu, and P. Maletinsky, New J. Phys. 17, 112001 (2015).

${ }^{30}$ J. A. Sedlacek, A. Schwettmann, H. Kübler, R. Löw, T. Pfau, and J. P. Shaffer, Nat. Phys. 8, 819 (2012).

${ }^{31}$ C. Holloway and J. Gordon, Appl. Phys. Lett. 104, 244102 (2014).

${ }^{32}$ H. Q. Fan, S. Kumar, R. Daschner, H. Kübler, and J. P. Shaffer, Opt. Lett. 39, 3030 (2014).

${ }^{33}$ H. Fan, S. Kumar, J. Sedlacek, H. Kübler, S. Karimkashi, and J. P. Shaffer, J. Phys. B 48, 202001 (2015).

${ }^{34}$ D. A. Steck, see http://steck.us/alkalidata for Rubidium 87 D Line Data (version 2.1.4, last revised 23 December 2010).

${ }^{35}$ See supplementary material at http://dx.doi.org/10.1063/1.4950805 for further details on the experiment and data analysis, as well as discussions of hyperfine transitions in arbitrary external dc magnetic fields, and the reconstruction of microwaves of arbitrary frequency.

${ }^{36}$ M. Pellaton, C. Affolderbach, Y. Pétremand, N. de Rooij, and G. Mileti, Phys. Scr. T149, 014013 (2012).

${ }^{37}$ Brukner, B-E 10 solenoid with B-MN C4 power supply, built in 1974.

${ }^{38}$ R. Mhaskar, S. Knappe, and J. Kitching, Appl. Phys. Lett. 101, 241105 (2012).

${ }^{39}$ M. A. Zentile, J. Keaveney, L. Weller, D. J. Whiting, C. S. Adams, and I. G. Hughes, Comput. Phys. Commun. 189, 162 (2015).

${ }^{40}$ A. Horsley, Ph.D. thesis (Department of Physics, University of Basel, Switzerland, 2015).

${ }^{41}$ A. Horsley, G.-X. Du, M. Pellaton, C. Affolderbach, G. Mileti, and P. Treutlein, in Proceedings of the 2013 Joint IEEE-UFFC, EFTF and PFM Symposium (2013), p. 575.

${ }^{42}$ S. Kadlecek, T. Walker, D. K. Walter, C. Erickson, and W. Happer, Phys. Rev. A 63, 052717 (2001).

${ }^{43}$ L. Weller, K. S. Kleinbach, M. A. Zentile, S. Knappe, I. G. Hughes, and C. S. Adams, Opt. Lett. 37, 3405 (2012)

${ }^{44}$ M. A. Zentile, R. Andrews, L. Weller, S. Knappe, C. S. Adams, and I. G. Hughes, J. Phys. B 47, 075005 (2014). 